\section{C.V. Raman on acoustics}

IN this, the third of four extracts from Raman's letters on acoustics selected to mark the author's centenary, Raman shows his skill in interpreting complicated experimental data. The figures he presents are of a type now well known as many perform similar studies of the behaviour of metals and plastics under impact. In Raman's day, such work was rare and he shows considerable insight in interpreting his Percussion Figures in Isotropic Solids. The reference to the work of Hertz is of interest because this work is usually overshadowed by his studies of electromagnetic phenomena.

J.M. Bowsher, University of Surrey

The accompanying photographs are of interest as illustrating the manner in which an isotropic solid breaks down under the stresses set up by impact when these exceed the limits of perfect recovery, and have a bearing on the theory of the collision of elastic solids developed by Hertz.

Figs 1, 2 and 3 are pictures of the percussion figure, taken from three different points of view, produced on the surface of a thick glass plate by

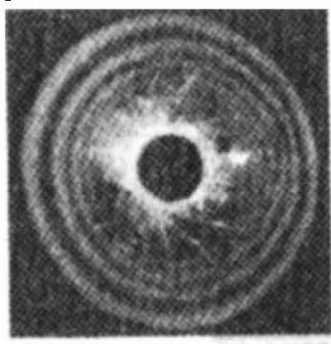

Fig. 1

Fig. 2
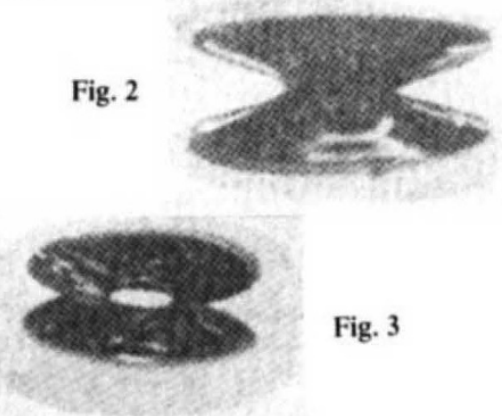

Fig. 3

the impact of a polished hard steel ball. Near the centre of the region of contact between the sphere and the plate the stresses are mainly in the nature of a volume-compression, and fracture accordingly does not originate there, but occurs at or near the margin of the compressed area in the form of a fine circular crack which spreads inwards into the plate obliquely in the form of a surface of revolution. This is clearly shown in Fig. 1, which is a front view of the percussion figure by reflected light, the dark circle in the middle being the uninjured area of contact between ball and plate. The circular interference-rings seen in the picture are a measure of the separation of the surfaces of the internal fracture within the plate.

Fig. 2 is a side view, and Fig. 3 an oblique view, of the internal fracture seen through the edge of the plate, the lower half of each picture being the image of the upper half formed by the reflection of light at the interior surface of the plate. The circular area of contact from the margin of which the fracture starts appears in Fig. 3 as an elliptic white disc at the centre. It seems clear that the internal fracture practically occurs along the surface of maximum shearing stress set up during the impact.

From Nature 104, 113 (1919).

\title{
Ecological genetics
} \section{8 and all that for Magicicada}

\author{
Godfrey M. Hewitt, Richard A. Nichols and Michael G. Ritchie
}

ON page 237 of this issue ${ }^{1}$, Martin and Simon describe the use of mitochondrial DNA markers to unravel the evolutionary origins of periodical cicadas of the genus Magicicada. Martin and Simon suggest that an ecological factor caused an extraordinary concerted change from a life cycle of 17 years to one of 13 years throughout a region including much of Illinois and Missouri. If changes in generation time do occur as the authors suggest, their results will help to explain the complex pattern of emergence of these insects and the evolution of their uniquely long life cycles.

There are three species of Magicicada living underground on roots in the deciduous forest region of the eastern United States. In the Mississippi valley and southern states, their life cycle is 13 years, whereas to the north and west it is 17 years. Nearly every year, in some part of the range, adult cicadas emerge synchronously in huge numbers: populations of 1.5 million individuals per acre have been recorded ${ }^{2}$. Cicadas with the same life cycle emerging in the same year are designated a brood. Thirteen of the possible 17 broods of the 17-year cicadas, and three of the 13 possible 13-year broods, have been identified. This synchronized emergence may be advantageous in evolutionary terms because the large numbers satiate predators ${ }^{3}$, and the long life cycles may prevent predator population sizes becoming synchronized with the emergence time. But if there is strong selection against non-synchronizing individuals, it is difficult to imagine how the life-cycle shift from 17 to 13 years could have happened ${ }^{+}$.

There are records of the emergence of these populations of periodic cicadas going back for more than 100 years. There are reports of small emergences out of step, and evidence that "4-year accelera- tions' have taken place, in which broods with the same cycle length occur 4 years apart in the same woods. It is interesting that the development of the 17-year cicada involves a 4-year early nymphal period when growth is inhibited; elimination of this period would produce a 13-year life cycle. In Illinois, there is good historical evidence for the disappearance of a 17year brood and the emergence of a new 13-year $\operatorname{brood}^{2}$ (see figure). The emergence dates of these two broods coincide in 1868 . Furthermore, the 17-year brood X and the 13-year brood XIX both occur in Illinois, an observation prompting Lloyd et al. to suggest ${ }^{5}$ that hybridization between the broods caused the change in the life cycle. The appearance of a small brood in 1898 is consistent with the idea that a single gene controls life cycle: this brood could consist of second-generation $\left(\mathrm{F}_{2}\right)$ segregants $(1868+13+17=1898)$.

Martin and Simon in this issue show that the 13-year brood in the Illinois area has mitochondrial DNA like that in the adjacent 17-year brood $X$, and distinct from that of the neighbouring 13-year brood XIX. Furthermore, the new brood is like the 17-year brood in abdominal colour and frequency of the enzyme phosphoglucomutase (PGM). (This enzyme is one of three polymorphic enzymes analysed by Martin and Simon to provide information on phylogenetic relationships.) Consequently, Martin and Simon argue for an environmentally induced switch in life cycle from 17 to 13 years, and they suggest that high population density may have triggered this switch.

The observed changes in life cycle could therefore result from either hybridization involving dominance for a 13-year cycle, as Lloyd and collaborators suggest, or from a density-induced 4-year acceleration, as suggested by Martin and Simon. There are problems with both

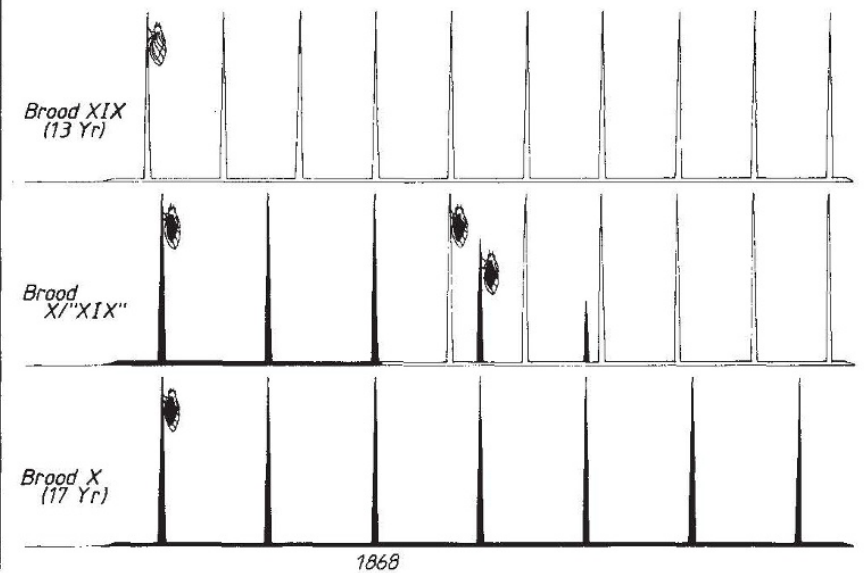

Brood XIX (13-year cycle) to the south emerged together with brood $X(17$ year cycle) from the north in 1868 , and in the region of overlap a 13-year brood replaced the 17-year brood. Dark individuals represent broods with colouring, $P G M$ frequency and mitochondrial DNA typical of brood $X$. 